\title{
Abietane and nor-abitane diterpenoids from the roots of Salvia rhytidea
}

\author{
Farrokh Eghtesadi ${ }^{1}$, Mahdi Moridi Farimani ${ }^{2 *}$, Nourallah Hazeri ${ }^{{ }^{*}}$ and Jafar Valizadeh ${ }^{3}$
}

\begin{abstract}
Background: The genus Salvia is a rich source of structurally diverse terpenoids. Different species of the Salvia have been used in folk medicine of Iran and therefore attracted the attention of researchers for exploring their chemical constituents. In a project directed at structurally interesting bioactive metabolites from Iranian Lamiaceae, we studied Salvia rhytidea.

Results: Fractionation of the petroleum ether extract of the root of S. rhytidea led to the isolation of a new 20-norabietane diterpenoid (1), together with seven known compounds, comprising five abietane diterpenoids (2-6), and two rearranged abietanes $(\mathbf{7}, \mathbf{8})$. Their structures were established by a combination of 1D and 2D NMR.

Conclusions: Our results showed that the root of $S$. rhytidea could be considered as a new and rich source of different types of abietane and rearranged abietane diterpenoids.

Keywords: Salvia rhytidea, Diterpenoid, Abietane, Solvent extraction, Column chromatography, Structure elucidation
\end{abstract}

\section{Background}

The genus Salvia is a rich source of structurally diverse terpenoids (Kintzios 2000; Moridi Farimani et al. 2013). Among these, numerous diterpenoids with promising bioactivities, such as antileishmanial, antitumor, antimicrobial, antifungal properties, have been reported from Salvia species (Ebrahimi et al. 2013; Tan et al. 2002; Akaberi et al. 2015; Moridi Farimani and Miran 2014; Ulubelen 2003; Jassbi et al. 2006). The most abundant diterpenoids in the genus are abietanes and rearranged abietanes (Wu et al. 2012). The genus Salvia is represented in the Iranian flora by 61 species, of which 17 are endemic (Jamzad et al. 2012). Salvia rhytidea Benth is an endemic species that grows widely in the eastern parts of Iran (Rechinger 1987). In our efforts to discover new and potentially bioactive secondary metabolites from Iranian Salvia species (Moridi Farimani and Mazarei 2014; Ebrahimi et al. 2014; Moridi Farimani et al. 2012; Bahadori

\footnotetext{
*Correspondence: m moridi@sbu.ac.ir; nhazeri@chem.usb.ac.ir ${ }^{1}$ Department of Chemistry, Faculty of Science, University of Sistan and Baluchestan, Zahedan, P.O. box 98135-674, Iran

2 Department of Phytochemistry, Medicinal Plants and Drugs Research Institute, Shahid Beheshti University, G. C., Evin, Tehran, Iran Full list of author information is available at the end of the article
}

et al. 2015; Moridi Farimani et al. 2008), we investigated the petroleum ether extract of the root of S. rhytidea. Here we report the isolation and structure elucidation of 1-deoxo aurocadiol (1), as a new 20-nor-abietane diterpenoid. In addition, the abietane diterpenoids ferruginol (2), taxodione (3), arucadiol (4), deoxyneocryptotanshinone (5), and $7 \alpha$ - Ethoxyroyleanone (6), and rearranged abietanes microstegiol (7) and 12-hydroxysapriparaquinone (8) were isolated and are described here for S. rhytidea for the first time.

\section{Results and discussion}

Compound 1 (Fig. 1) was isolated as an orange, amorphous solid. The IR spectrum showed absorptions of hydroxy $\left(3475 \mathrm{~cm}^{-1}\right)$ and olefinic $\left(1610 \mathrm{~cm}^{-1}\right)$ functionalities. The ${ }^{13} \mathrm{C}$ NMR spectrum showed 19 carbon resonances, which were identified with the aid of HSQC and DEPTQ spectra as four methyl, three methylene, four methine, and eight quaternary carbons. The ${ }^{13} \mathrm{C}$ NMR spectrum showed signals indicative of ten aromatic carbons. The ${ }^{1} \mathrm{H}$ NMR spectrum showed resonances of two methyl singlets at $\delta_{\mathrm{H}} 1.22(\mathrm{~s}, 6 \mathrm{H})$. Resonances of two additional methyl groups at $\delta_{\mathrm{H}} 1.14(\mathrm{~d}, J=6.9 \mathrm{~Hz})$ and $1.19(\mathrm{~d}, J=6.9 \mathrm{~Hz})$, together with a signal at $\delta_{\mathrm{H}} 2.90$ 
<smiles>CC(I)c1cc2ccc3c(c2c(O)c1O)CCCC3(C)C</smiles>

1<smiles>CC(C)c1cc2c(cc1O)[C@]1(C)CCCC(C)(C)C1CC2</smiles>

2<smiles>CC(C)C1=CC2=CC(=O)C3C(C)(C)CCC[C@]3(C)C2=C(O)C1=O</smiles><smiles>CC(C)c1cc2ccc3c(c2c(O)c1O)C(=O)CCC3(C)C</smiles>

4<smiles>CC(C)C1=C(O)C(=O)c2c(ccc3c2CCCC3(C)C)C1=O</smiles>

5<smiles>CCO[C@H]1CC2C(C)(C)CCC[C@]2(C)C2=C1C(=O)C(C(C)C)=C(O)C2=O</smiles>

6<smiles>Cc1ccc2c3c1CCC[C@](C)(O)[C@]3(O)C(=O)C(C(C)C)=C2</smiles>

7<smiles>CC(C)=CCCc1c(C)ccc2c1C(=O)C(O)=C(C(C)C)C2=O</smiles>

8

Fig. 1 Structure of compounds 1-8

(sept, $J=6.9 \mathrm{~Hz}$ ) indicated the presence of an isopropyl moiety. Signals at $\delta_{\mathrm{H}} 7.02(\mathrm{~d}, J=7.0 \mathrm{~Hz})$ and 7.43 $(\mathrm{d}, J=7.0 \mathrm{~Hz})$ were indicative of two aromatic protons with ortho-position to each other. Another aromatic methine signal appeared as a singlet at $\delta_{\mathrm{H}} 6.78$. Therefore, the structural features were reminiscent of a norabietane diterpenoid containing two aromatic rings. The NMR data of $\mathbf{1}$ showed great similarity to those of arucadiol isolated from S. argentea (Michavila et al. 1986) and S. miltiorrhiza (Ginda et al. 1988). Inspection of the ${ }^{13} \mathrm{C}$ NMR spectra showed the lack of a carbonyl group in compound 1 but the presence of an additional methylene group at $\delta_{\mathrm{C}} 27.6$. The chemical shifts of C-2, C-5, and C-7 were observed at $\delta_{\mathrm{C}} 18.5,148.8$, and 126.5, respectively, with upfield shifts of ca. 18,10 , and $11 \mathrm{ppm}$ relative to arucadiol, while the chemical shift of C-3, C-6, C-9, and C-10 were observed at $\delta_{\mathrm{C}} 38.0,129.8,128.4$, and 134.6 with downfield shift of ca. 3, 10, 9, and $10 \mathrm{ppm}$, respectively. These observations suggested the replacement of the $\mathrm{C}-1$ carbonyl with a methylene group. HMBC correlations (Fig. 2) between $\mathrm{H}-1\left(\delta_{\mathrm{H}} 2.78,2 \mathrm{H}, t\right)$, and $\mathrm{C}-2$ $\left(\delta_{\mathrm{C}} 18.5\right), \mathrm{C}-5\left(\delta_{\mathrm{C}} 148.8\right), \mathrm{C}-10\left(\delta_{\mathrm{C}} 134.6\right)$, and C-9 $\left(\delta_{\mathrm{C}}\right.$ 128.4), and COSY correlation between $\mathrm{H}-1$ and $\mathrm{H}-2\left(\delta_{\mathrm{H}}\right.$ $1.72,2 \mathrm{H}, \mathrm{m})$ confirmed the location of the methylene group. Unambiguous assignments of NMR data were achieved by a combination of COSY, HMQC, and HMBC 


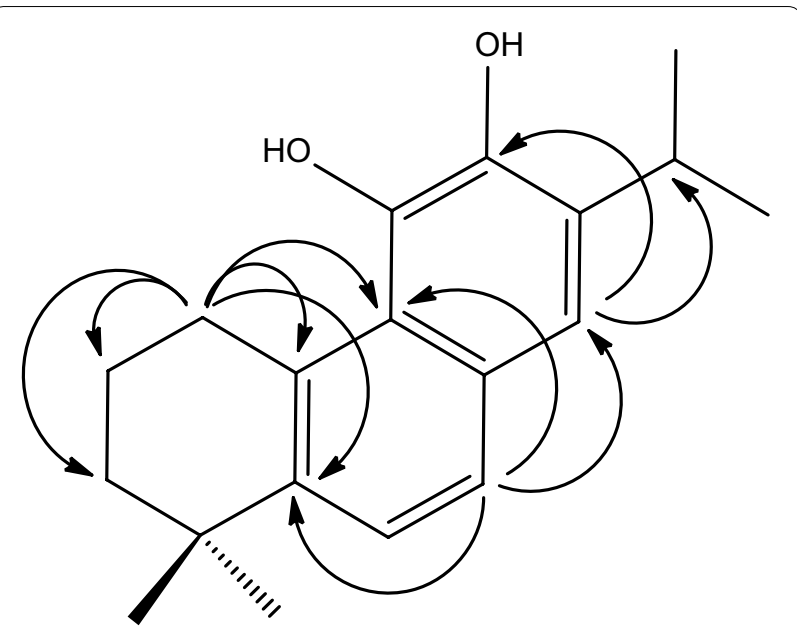

Fig. 2 Key HMBC correlations of 1

experiments. Compound $\mathbf{1}$ was therefore established structurally as 1-deoxo-arucadiol.

Ferruginol (2) is a well known abietane diterpenoid which was isolated from different Salvia species such as S. syriaca and S. sclarea (Ulubelen et al. 2000, 1994). Antileishmanial (Tan et al. 2002), antimicrobial (Ulubelen et al. 1999), cytotoxic (Moujir et al. 1996; Fronza et al. 2011), antihypertensive (Ulubelen et al. 1994), and anticholinesterase (Topcu et al. 2013) activities were reported for ferruginol and the mechanism of its antioxidant properties was also investigated (Saijo et al. 2015).

Taxodione (3) is a diterpenoid with quinone methide skeleton which was reported from different genus like Taxudium, Clerodendrum, and Salvia (Machumi et al. 2010; Kolak et al. 2009; Kusumoto et al. 2009). Different biological properties have been reported for this compound, including antibacterial (Yang et al. 2001), antioxidant (Kolak et al. 2009), antitermitic (Kusumoto et al. 2009), antifeedant (Acosta et al. 2008), antifungal (Topçu and Gören 2007), and anticholinesterase activities (Topcu et al. 2013). Moreover, cytotoxic and tumor inhibitory properties of taxodione have been investigated in in situ and in vivo experiments (Kupchan et al. 1969; Ulubelen et al. 1999; Abou Dahab et al. 2007). The mechanism of action of taxodione for its cytotoxic properties was investigated in several articles with focus on its DNA binding and DNA damaging character (Zaghloul et al. 2008), and its enzyme inhibitory action (Hanson et al. 1970).

Arucadiol (miltiodiol) (4) was previously extracted from S. argentea (Michavila et al. 1986), S. miltiorrhiza (Ginda et al. 1988), S. prionitis (Yong 1995), and S. apiana (González et al. 1992). Cytotoxic properties have been reported for arucadiol (Fronza et al. 2011).
Deoxyneocryptotanshinone (5) is a para-quinone abitanne diterpenod with cytotoxic activity which was isolated from S. miltiorrhiza for the first time (Ikeshiro et al. 1991).

$7 \alpha$-Ethoxyroyleanone (6) has been reported from $S$. lavandulaefolia, S. lanigra, and Peltodon longipes and its cytotoxic and antioxidant effects were investigated (Fronza et al. 2011; Shaheen et al. 2011; Michavila et al. 1985; Burmistrova et al. 2013).

Microstegiol (7) is a rearranged abietane with a sevenmember ring skeleton. This compound has solely been reported from Salvia genus so far (Topcu et al. 2013; Ulubelen et al. 1992). It has been shown to have mild antibacterial effects (Topçu and Gören 2007).

12-hydroxysapriparaquinone (8), a rearranged 4,5-seco-abietane diterpenoid, previously isolated from $S$. limbata (Topcu et al. 1996).

\section{Experimental}

\section{General experimental procedures}

NMR spectra were recorded at a target temperature of $18{ }^{\circ} \mathrm{C}$ on a Bruker Avance III $500 \mathrm{MHz}$ spectrometer operating at $500.13 \mathrm{MHz}$ for ${ }^{1} \mathrm{H}$ and $125.77 \mathrm{MHz}$ for ${ }^{13} \mathrm{C}$. 2D NMR experiments $\left({ }^{1} \mathrm{H}^{-1} \mathrm{H}\right.$ COSY, HSQC, HMBC, NOESY) were performed using Bruker microprograms. $\mathrm{CDCl}_{3}$ was purchased from Armar Chemicals. TLC was performed on silica gel (Merck, Kieselgel 60, $\mathrm{F}_{254}$, $0.25 \mathrm{~mm}$ ) phase. Column chromatography $(\mathrm{CC})$ was carried out using silica gel (70-230 mesh, Merck). Flash column chromatography (FCC) was performed on silica gel (230-400 mesh, Merck).

\section{Plant material}

The roots of Salvia rhytidea were collected from Taftan Mountain, $28^{\circ} 36^{\prime} \mathrm{N}$ and $61^{\circ} 4^{\prime} \mathrm{E}$, in the Baluchistan of Iran at an altitude of $2497 \mathrm{~m}$, in autumn 2012. The plant was authenticated by Dr. Valizadeh and a voucher specimen (no. 4938) was deposited in the Herbarium of the School of Biology (Dr. Akhani Herbarium), University of Tehran.

\section{Extraction and purification}

The air-dried roots of S. rhytidea (2100 g) were crushed and extracted with petroleum ether (bp. $40-60{ }^{\circ} \mathrm{C}$ ) at room temperature $\left(25^{\circ} \mathrm{C}\right)$ for 5 days. The extract was concentrated in vacuo, to afford $12.6 \mathrm{~g}$ dark gummy residue. It was subjected to a silica gel column chromatography using petroleum ether $(3 \mathrm{~L})$ as eluting solvent, increasing the polarity with a mixture of dichloromethane: acetone (90:10, 5 L), to yield nine fractions (1-9) (Fig. 3). Fraction 3 was subjected to silica gel flash column chromatography using petroleum ether-diethyl ether $(98: 2,2.5 \mathrm{~L})$, as 


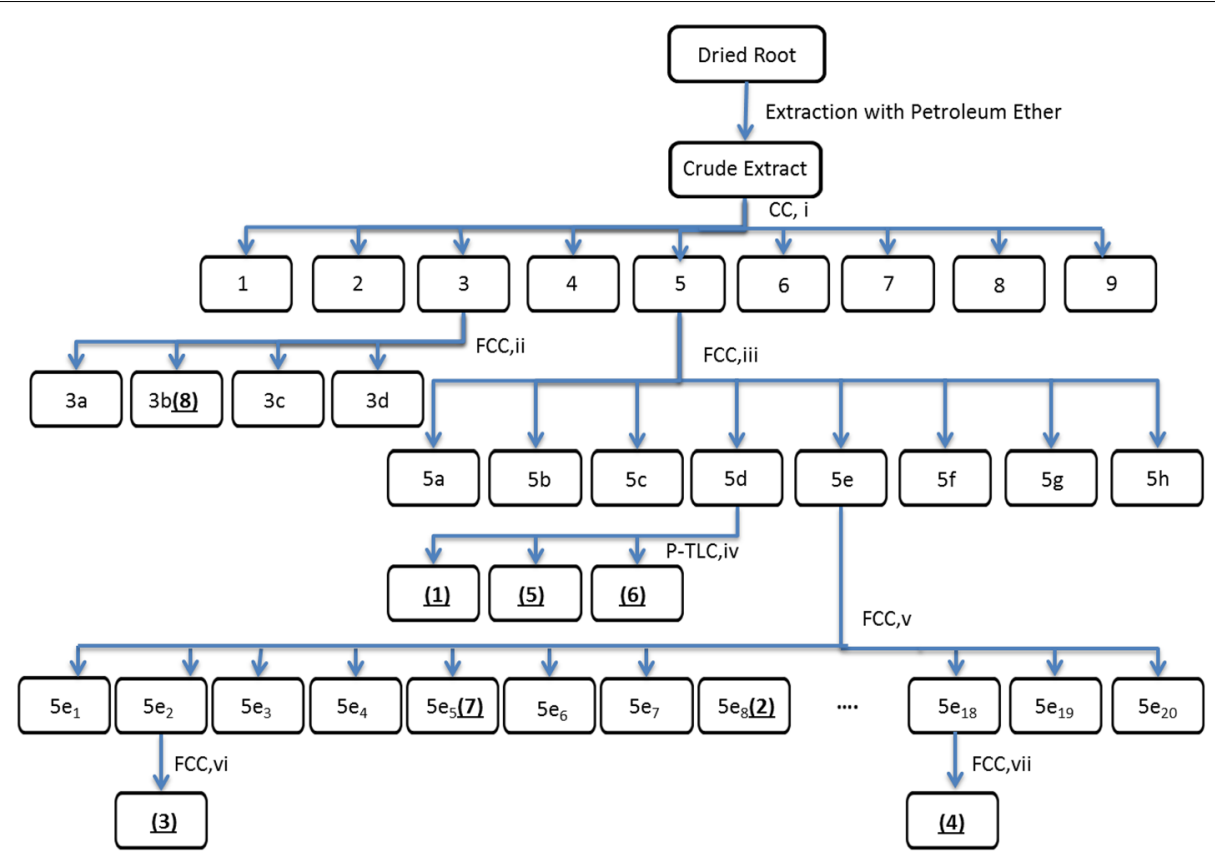

Fig. 3 Schematic diagram of extraction and purification procedure for petroleum ether extract of S. rhytidea roots

mobile phase. Four subfractions (3a-3d) were collected. Subfraction 3b was recrystallized from $\mathrm{Me}_{2} \mathrm{CO}$ to afford $8(8 \mathrm{mg})$. Fraction 5 was separated on a silica gel flash column with a gradient of petroleum ether-EtOAc (100:0, $0.5 \mathrm{~L} ; 90: 10,0.3 \mathrm{~L}, 80: 20,0.3 \mathrm{~L} ; 70: 30,0.3 \mathrm{~L} ; 60: 40,0.2 \mathrm{~L}$; $50: 50,0.2 \mathrm{~L})$ as eluent, to yield 8 subfractions $(5 \mathrm{a}-5 \mathrm{~h})$. Subfraction $5 \mathrm{~d}$ was purified by preparative TLC [developed with acetone-EtOAc-petroleum ether (2:2:96)] to afford 1 (4 mg), 5 (5 mg), and 6 (5 mg). Subfraction $5 \mathrm{e}$ was separated over a silica gel flash column, eluting with petroleum ether- $\mathrm{CH}_{2} \mathrm{Cl}_{2}(85: 15,1 \mathrm{~L})$ followed by increasing concentrations of $\mathrm{CH}_{2} \mathrm{Cl}_{2}$ (up to $100 \%$ ), to afford 20 subfractions $\left(5 \mathrm{e}_{1}-5 \mathrm{e}_{20}\right)$. Compounds $7(10 \mathrm{mg})$ and 2 (7 mg) were recrystallized from subfractions $5 \mathrm{e}_{5}$ and $5 \mathrm{e}_{8}$, respectively. Subfraction $5 e_{2}$ was purified by another silica gel column to yield $3(5 \mathrm{mg})$. Subfraction $5 \mathrm{e}_{18}$ was also separated on another silica gel column to yield 4 ( $3 \mathrm{mg}$ ).

\section{1-Deoxoarucadiol (1)}

${ }^{1} \mathrm{H}-\mathrm{NMR}\left(500 \mathrm{MHz}\right.$, in $\left.\mathrm{CDCl}_{3}\right): \delta 1.14(3 \mathrm{H}, \mathrm{d}, \mathrm{J}=6.9 \mathrm{~Hz}$, Me-16), $1.19(3 \mathrm{H}, \mathrm{d}, \mathrm{J}=6.9 \mathrm{~Hz}, \mathrm{Me}-17), 1.22(6 \mathrm{H}, \mathrm{s}$, Me-18 and Me-19), 1.58 (2H, m, H-3), 1.72 (2H, m, H-2), $2.78(2 \mathrm{H}, \mathrm{t}, \mathrm{J}=6.3 \mathrm{~Hz}, \mathrm{H}-1), 2.90(1 \mathrm{H}$, sept., $\mathrm{J}=6.9 \mathrm{~Hz}$, $\mathrm{H}-15), 6.78(1 \mathrm{H}, \mathrm{s}, \mathrm{H}-14), 7.02(1 \mathrm{H}, \mathrm{d}, \mathrm{J}=7.0 \mathrm{~Hz}, \mathrm{H}-7)$, $7.43(1 \mathrm{H}, \mathrm{d}, \mathrm{J}=7.0 \mathrm{~Hz}) ;{ }^{13} \mathrm{C}-\mathrm{NMR}\left(125 \mathrm{MHz}\right.$, in $\left.\mathrm{CDCl}_{3}\right)$ : 18.5 (C-2), 20.6 (C-16 and C-17), 27.6 (C-1), 31.8 (C-18 and C-19), 32.4 (C-15), 34.0 (C-4), 38.0 (C-3), 126.5 (C-7), 128.4 (C-9), 129.8 (C-6), 131.6 (C-14), 134.6 (C-10), 139.7
(C-13), 140.4 (C-8), 148.8 (C-5), 152.9 (C-11), 161.2 (C-12).

\section{Ferruginol (2)}

${ }^{1} \mathrm{H}-\mathrm{NMR}\left(500 \mathrm{MHz}\right.$, in $\mathrm{CDCl}_{3}$ ); $\delta 0.92$ and 0.95 (each $3 \mathrm{H}$, s, Me-18 and Me-19), 1.17 (3H, s, Me-20), $1.22(1 \mathrm{H}, \mathrm{m}$, $\mathrm{H}-3$ ), 1.25 and 1.26 (each $3 \mathrm{H}, \mathrm{d}, \mathrm{J}=7.0 \mathrm{~Hz}, \mathrm{Me}-16$ and Me-17), $1.33(1 \mathrm{H}, \mathrm{d}, \mathrm{J}=12.0$ and $2.2 \mathrm{~Hz}, \mathrm{H}-5), 1.37(1 \mathrm{H}$, $\mathrm{m}, \mathrm{H}-1), 1.48(1 \mathrm{H}, \mathrm{m}, \mathrm{H}-3) 1.58(1 \mathrm{H}, \mathrm{dt}, \mathrm{J}=13.9$ and $3.4 \mathrm{~Hz}, \mathrm{H}-2), 1.68(1 \mathrm{H}$, brd, J = $12.9 \mathrm{~Hz}, \mathrm{H}-6), 1.72(1 \mathrm{H}$, $\mathrm{m}, \mathrm{H}-2), 1.88(1 \mathrm{H}, \mathrm{tdd}, \mathrm{J}=13.2,7.4$, and $1.8 \mathrm{~Hz}, \mathrm{H}-6)$, $2.14(1 \mathrm{H}, \mathrm{brd}, \mathrm{J}=12.5 \mathrm{~Hz}, \mathrm{H}-1), 2.80(1 \mathrm{H}, \mathrm{dd}, \mathrm{J}=11.4$ and $7.4 \mathrm{~Hz}, \mathrm{H}-7), 2.88(1 \mathrm{H}$, ddd, $\mathrm{J}=11.4$ and $7.4 \mathrm{~Hz}$, H-7), 3.20 (1H, sept., J = 6.9 Hz, H-15), 5.34 (s, OH), 6.69 (1H, s, H-11), 6.86 (1H, s, H-14), ${ }^{13} \mathrm{C}-\mathrm{NMR}(125 \mathrm{MHz}$, in $\left.\mathrm{CDCl}_{3}\right): \delta 20.1(\mathrm{C}-2), 20.4(\mathrm{C}-6), 22.7(\mathrm{C}-19), 23.7$ (C-16), 23.8 (C-17), 26.2 (C-20), 27.7 (C-15), 31.0 (C-7), 33.4 (C-18), 34.5 (C-4), 38.8 (C-10), 40.0 (C-1), 42.8 (C-3), 51.5 (C-5), 111.8 (C-11), 127.5 (C-14), 127.8 (C-8), 132.5 (C-13), 149.5 (C-9), 151.8 (C- 12).

\section{Taxodione (3)}

${ }^{1} \mathrm{H}-\mathrm{NMR}$ (500 MHz, in $\mathrm{CDCl}_{3}$ ): $\delta 1.18$ and 1.21 (each $3 \mathrm{H}, \mathrm{d}, \mathrm{J}=6.9 \mathrm{~Hz}, \mathrm{Me}-16$ and Me-17), 1.15, 1.30 and 1.30 (each $3 \mathrm{H}, \mathrm{s}, \mathrm{Me}-18, \mathrm{Me}-19$ and Me-20), 1.20 and 1.44 $(1 \mathrm{H}, \mathrm{m}, \mathrm{H}-3), 1.67$ and 1.86 (each $1 \mathrm{H}, \mathrm{m}, \mathrm{H}-2), 2.64(1 \mathrm{H}$, brs, H-5), $2.97(1 \mathrm{H}$, ddd, $\mathrm{J}=10.7,3.6$ and $1.3 \mathrm{~Hz}, \mathrm{H}-1)$, $3.10(1 \mathrm{H}$, dsept., $\mathrm{J}=6.9$ and $0.8 \mathrm{~Hz}, \mathrm{H}-15), 6.26(1 \mathrm{H}, \mathrm{s}$, 
H-7), 6.91 (1H, brs, H-14), 7.66 (s, 11-OH); ${ }^{13} \mathrm{C}-\mathrm{NMR}$ $\left(125 \mathrm{MHz}\right.$, in $\left.\mathrm{CDCl}_{3}\right): \delta 19.3(\mathrm{C}-2), 22.7(\mathrm{C}-20), 22.7$ (C-16), 22.8 (C-17), 22.8 (C-19), 28.1 (C- 15), 33.0 (C-4), 33.6 (C-18), 38.3 (C-1), 42.7 (C-3), 43.5 (C-10), 63.1 (C-5), 126.7 (C-9), 135.1 (C-7), 137.5 (C-14), 140.0 (C-8), 145.1 (C-11), 146.2 (C- 13), 182.7 (C-12), 201.7 (C-6).

\section{Arucadiol (4)}

${ }^{1} \mathrm{H}-\mathrm{NMR}\left(500 \mathrm{MHz}\right.$, in $\left.\mathrm{CDCl}_{3}\right) ; \delta 1.37(6 \mathrm{H}, \mathrm{d}, \mathrm{J}=6.9 \mathrm{~Hz}$ Me-16 and Me-17), 1.47 (6H, s, Me-18 and Me-19), 2.09 $(2 \mathrm{H}, \mathrm{t}, \mathrm{J}=6.8 \mathrm{~Hz}, \mathrm{H}-3), 2.94(2 \mathrm{H}, \mathrm{t}, \mathrm{J}=6.8 \mathrm{~Hz}, \mathrm{H}-2)$, 3.47 (1H, sept., J = $6.9 \mathrm{~Hz}, \mathrm{H}-15), 6.89$ (brs, 11-OH), 7.28 $(1 \mathrm{H}, \mathrm{s}, \mathrm{H}-14), 7.33(1 \mathrm{H}, \mathrm{d}, \mathrm{J}=8.6 \mathrm{~Hz}, \mathrm{H}-6), 7.95(1 \mathrm{H}, \mathrm{d}$, $\mathrm{J}=8.6 \mathrm{~Hz}, \mathrm{H}-7), 10.73$ (s, 11-OH), ${ }^{13} \mathrm{C}-\mathrm{NMR}(125 \mathrm{MHz}$, in $\left.\mathrm{CDCl}_{3}\right): \delta 22.0(\mathrm{C}-16$ and $\mathrm{C}-17), 27.4(\mathrm{C}-15), 29.6$ (C-18 and C-19), 35.5 (C-3), 36.2 (C-2), 118.5 (C-14), 120.0 (C-11), 120.1 (C-6), 126.7 (C-10), 127.7 (C-9), 128.4 (C-8), 136.6 (C-13), 137.8 (C-7), 148.2 (C-12), 158.3 (C-5), $204.3(\mathrm{C}-1)$.

\section{Deoxyneocryptotanshinone (5)}

${ }^{1} \mathrm{H}-\mathrm{NMR}\left(500 \mathrm{MHz}\right.$, in $\left.\mathrm{CDCl}_{3}\right): \delta 1.29(6 \mathrm{H}, \mathrm{d}, \mathrm{J}=7.0 \mathrm{~Hz}$, Me-16 and Me-17), 1.30 (6H, s, Me-18 and Me-19), 1.79 $(4 \mathrm{H}, \mathrm{m}, \mathrm{H}-2$ and $\mathrm{H}-3), 3.20(2 \mathrm{H}, \mathrm{t}, \mathrm{J}=6.4 \mathrm{~Hz}), 3.37(1 \mathrm{H}$, sept., J = 6.5 Hz, H-15), $7.71(1 \mathrm{H}, \mathrm{d}, \mathrm{J}=8.2 \mathrm{~Hz}, \mathrm{H}-6)$, $7.97(1 \mathrm{H}, \mathrm{d}, \mathrm{J}=8.2 \mathrm{~Hz}, \mathrm{H}-7), 7.82(1 \mathrm{H}, \mathrm{s}, 12-\mathrm{OH}) ;{ }^{13} \mathrm{C}-$ NMR (125 MHz, in $\left.\mathrm{CDCl}_{3}\right)$ : 19.2 (C-2), 19.9 (C-16 and C-17), 24.5 (C-15), 30.0 (C-1), 31.8 (C-18 and C-19), 37.8 (C-3), 123.8 (C-13), 125.1 (C-7), 126.5 (C-9), 132.8 (C-8), 133.4 (C-6), 140.7 (C-4), 152.5 (C-10), 153.3 (C-12), 183.4 (C-11), 184.6 (C-14).

\section{7a-Ethoxyroyleanone (6)}

${ }^{1} \mathrm{H}-\mathrm{NMR}\left(500 \mathrm{MHz}\right.$, in $\left.\mathrm{CDCl}_{3}\right): \delta 0.84$ and 0.87 (each $3 \mathrm{H}$, s, Me-18 and Me-19), 1.11 (3H, t, J = 5.9 Hz, Me-2'), 1.12 and 1.15 (each $3 \mathrm{H}, \mathrm{d}, \mathrm{J}=7.1 \mathrm{~Hz}, \mathrm{Me}-16$ and Me-17), 1.13 $(3 \mathrm{H}, \mathrm{s}, \mathrm{Me}-20), 1.13(1 \mathrm{H}$, overlap, $\mathrm{H}-1), 1.17(1 \mathrm{H}$, overlap, $\mathrm{H}-3), 1.29(1 \mathrm{H}, \mathrm{m}, \mathrm{H}-6), 1.39(1 \mathrm{H}, \mathrm{brd}, \mathrm{J}=13.1 \mathrm{~Hz}$, $\mathrm{H}-3), 1.47(1 \mathrm{H}, \mathrm{dt}, \mathrm{J}=14.0,3.5 \mathrm{~Hz}, \mathrm{H}-2), 1.57(1 \mathrm{H}$, $\mathrm{d}, \mathrm{J}=12.8 \mathrm{~Hz}, \mathrm{H}-8), 1.64(1 \mathrm{H}, \mathrm{m}, \mathrm{H}-2), 1.93(1 \mathrm{H}, \mathrm{d}$, $\mathrm{J}=14.1 \mathrm{~Hz}, \mathrm{H}-14), 2.60(1 \mathrm{H}, \mathrm{brd}, \mathrm{J}=13.1 \mathrm{~Hz}, \mathrm{H}-1), 3.10$ $(1 \mathrm{H}$, sept., $\mathrm{J}=7.1 \mathrm{~Hz}, \mathrm{H}-15), 3.61\left(2 \mathrm{H}, \mathrm{m}, \mathrm{H}-1^{\prime}\right), 4.35$ $(1 \mathrm{H}, \mathrm{dd}, \mathrm{J}=3.2$ and $1.6 \mathrm{~Hz}, \mathrm{H}-7) ;{ }^{13} \mathrm{C}-\mathrm{NMR}(125 \mathrm{MHz}$, in $\left.\mathrm{CDCl}_{3}\right)$ : $15.5\left(\mathrm{C}-2^{\prime}\right), 18.5(\mathrm{C}-2), 18.7$ (C-16 and $\left.\mathrm{C}-17\right)$, 18.8 (C-20), 21.5 (C-19), 22.8 (C-6), 23.4 (C-15), 32.5 (C-18), 33.0 (C-4), 35.2 (C-1), 38.9 (C-10), 40.5 (C-5), 45.2 (C-5), 65.0 (C-1'), 68.6 (C-7), 124.8 (C-13), 141.2 (C-8), 146.9 (C-9), 150.2 (C-12), 184.0 (C-11), 186.1 (C-14).

\section{Microstegiol (7)}

${ }^{1} \mathrm{H}-\mathrm{NMR}\left(500 \mathrm{MHz}\right.$, in $\mathrm{CDCl}_{3}$ ): $\delta 0.80$ and 0.81 (each $3 \mathrm{H}, \mathrm{s}, \mathrm{Me}-18$ and Me-19), 1.17 and 1.22 (each $3 \mathrm{H}, \mathrm{d}$. $\mathrm{J}=6.9 \mathrm{~Hz}, \mathrm{Me}-16$ and Me-17), 1.27 and 2.4 (each $1 \mathrm{H}, \mathrm{m}$,
$\mathrm{H}-3$ ), 1.42 and 1.69 (each $1 \mathrm{H}, \mathrm{m}, \mathrm{H}-2), 2.33$ (3H, s, H-20), $2.80(1 \mathrm{H}, \mathrm{ddd}, \mathrm{J}=14.0,6.2$ and $2.3 \mathrm{~Hz}, \mathrm{H}-1), 3.03(1 \mathrm{H}$, dsept, $J=6.7$ and $0.7 \mathrm{~Hz}, \mathrm{H}-15), 3.61(1 \mathrm{H}, \mathrm{dt}, \mathrm{J}=2.1$ and $13.1 \mathrm{~Hz}, \mathrm{H}-1), 4.53(\mathrm{~s}, 11-\mathrm{OH}), 6.91$ and $7.07(1 \mathrm{H}, \mathrm{d}$, $\mathrm{J}=7.6 \mathrm{~Hz}, \mathrm{H}-7$ and $\mathrm{H}-6), 6.97(1 \mathrm{H}, \mathrm{d}, \mathrm{J}=0.5 \mathrm{~Hz}, \mathrm{H}-14)$; ${ }^{13} \mathrm{C}-\mathrm{NMR}\left(125 \mathrm{MHz}\right.$, in $\left.\mathrm{CDCl}_{3}\right): 21.1$ (C-16), $21.4(\mathrm{C}-20)$, 21.5 (C-18), 22.1 (C-17), 23.5 (C-2), 26.9 (C-1), 27.1 (C-15), 28.0 (C-19), 39.0 (C-4), 42.9 (C-3), 84.3 (C-11), 126.7 (C-7), 129.1 (C-8), 130.1 (C-6), 137.3 (C-5), 139.3 (C-9), 140.4 (C-14), 141.0 (C-13), 143.3 (C-10), 206.18 (C-12).

\section{2-hydroxysapriparaquinone (8)}

${ }^{1} \mathrm{H}-\mathrm{NMR}\left(500 \mathrm{MHz}\right.$, in $\left.\mathrm{CDCl}_{3}\right): \delta 1.29(6 \mathrm{H}, \mathrm{d}, \mathrm{J}=7.0 \mathrm{~Hz}$, Me-16 and Me-17), 1.60 and 1.70 (each $3 \mathrm{H}, \mathrm{s}, \mathrm{Me}-18$ and Me-19), 1.65 (2H, m, H-3), 2.15 (2H, q, J = $8.2 \mathrm{~Hz}, \mathrm{H}-2$ ), $2.42(3 \mathrm{H}, \mathrm{s}, \mathrm{Me}-20), 3.16$ (2H, brt, J = 8.2 Hz, H-1), 3.37 $(1 \mathrm{H}$, sept., J $=6.5 \mathrm{~Hz}, \mathrm{H}-15), 5.27(1 \mathrm{H}, \mathrm{t}, \mathrm{J}=6.9 \mathrm{~Hz}, \mathrm{H}-3)$, $7.47(1 \mathrm{H}, \mathrm{d}, \mathrm{J}=7.9 \mathrm{~Hz}, \mathrm{H}-6), 7.83(1 \mathrm{H}, \mathrm{s}, 12-\mathrm{OH}), 7.93$ $(1 \mathrm{H}, \mathrm{J}=7.9 \mathrm{~Hz}, \mathrm{H}-7) ;{ }^{13} \mathrm{C}-\mathrm{NMR}\left(125 \mathrm{MHz}\right.$, in $\left.\mathrm{CDCl}_{3}\right)$ : 17.6 (C-19), 19.9 (C-18 and C-19), 20.4 (C-20), 24.6 (C-15), 27.1 (C-1), 30.3 (C-2), 38.5 (C-3), 124.7 (C-13), 125.5 (C-7), 126.2 (C-9), 132.8 (C-8), 136.3 (C-6), 143.3 (C-5), 144.4 (C-10), 145.6 (C-4), 153.3 (C-12), 183.4 (C-11), 184.5 (C-14).

\section{Conclusions}

Our results showed that the root of S. rhytidea contained different types of abietane and rearranged abietane diterpenoids. Biological properties of some of these compounds have been reported previously. Accordingly, $S$. rhytidea could be considered as a new and rich source of natural agents for the treatment of cancer, malaria, and microbial strains.

\section{Authors' contributions}

Analysis and interpretation of data: by FE, MM and $\mathrm{NH}$. All authors read and approved the final manuscript.

\section{Author details \\ 1 Department of Chemistry, Faculty of Science, University of Sistan and Bal- uchestan, Zahedan, P.O. box 98135-674, Iran. ${ }^{2}$ Department of Phytochemistry, Medicinal Plants and Drugs Research Institute, Shahid Beheshti University, G. C., Evin, Tehran, Iran. ${ }^{3}$ Department of Biology, Faculty of Science, University of Sistan and Baluchestan, Zahedan, P.O. box 98135-674, Iran.}

\section{Acknowledgements}

Financial support by the University of Sistan \& Baluchestan Research Council is gratefully acknowledged. All spectra were performed at the Department of Pharmaceutical Sciences, Division of Pharmaceutical Biology, University of Basel. The kind assistance of Prof. M. Hamburger, Dr. S.N. Ebrahimi, and all other staff is gratefully appreciated.

\section{Competing interests}

The authors declare that they have no competing interests.

Received: 1 May 2016 Accepted: 22 June 2016

Published online: 13 July 2016 


\section{References}

Abou Dahab MA, El-Bahr MK, Taha HS, Habib AM, Bekheet SA, Gabr AMM Refaat A (2007) Cytotoxic activity of Taxodium Calli Extracts on rat liver cells. J Appl Sci Res 3(12):1987-1996

Acosta MB, Villalobos MJP, Rodríguez B (2008) The antifeedant activity of natural plant products towards the larvae of Spodoptera littoralis. Span J agric Res 1:85-91

Akaberi M, Mehri S, Iranshahi M (2015) Multiple pro-apoptotic targets of abietane diterpenoids from Salvia species. Fitoterapia 100:118-132

Bahadori MB, Valizadeh H, Asghari B, Dinparast L, Moridi Farimani M, Bahadori S (2015) Chemical composition and antimicrobial, cytotoxicity, antioxidant and enzyme inhibitory activities of Salvia spinosa L. J Funct Foods 18:727-736

Burmistrova O, Simões MF, Rijo P, Quintana J, Bermejo J, Estévez F (2013) Antiproliferative activity of abietane diterpenoids against human tumor cells. J Nat Prod 76:1413-1423

Ebrahimi SN, Zimmermann S, Zaugg J, Smiesko M, Brun R, Hamburger M (2013) Abietane diterpenoids from Salvia sahendica-antiprotozoal activity and determination of their absolute configurations. Planta Med 79:150-156

Ebrahimi SN, Moridi Farimani M, Mirzania F, Soltanipoor MA, De Mieri M, Hamburger M (2014) Manoyloxide sesterterpenoids from Salvia mirzayanii. J Nat Prod 77:848-854

Fronza M, Murillo R, Ślusarczyk S, Adams M, Hamburger M, Heinzmann B, Laufer S, Merfort I (2011) In vitro cytotoxic activity of abietane diterpenes from Peltodon longipes as well as Salvia miltiorrhiza and Salvia sahendica. Bioorg med chem 19(16):4876-4881

Ginda H, Kusumi T, Ishitsuka MO, Kakisawa H, Weijie Z, Jun C, Tian GY (1988) Salviolone, a cytotoxic bisnorditerpene with a benzotropolone chromophore from a chinese drug dan-shen (Salvia miltiorrhiza). Tetrahedron Lett 29:4603-4606

González AG, Aguiar ZE, Grillo TA, Luis JG (1992) Diterpenes and diterpene quinones from the roots of Salvia apiana. Phytochemistry 31(5):1691-1695

Hanson RL, Lardy HA, Kupchan SM (1970) Inhibition of phosphofructokinase by quinone methide and a-methylene lactone tumor inhibitors. Science 168(3929):378-380

Ikeshiro Y, Hashimoto I, Iwamoto Y, Mase I, Tomita Y (1991) Diterpenoids from Salvia miltiorrhiza. Phytochemistry 30(8):2791-2792

Jamzad Z, Assadi M, Maassoumi A, Mozaffarian V (2012) Lamiaceae. In: Flora of Iran, vol. 76. Research Institute of Forest and Rangelands, Tehran

Jassbi AR, Mehrdad M, Eghtesadi F, Ebrahimi SN, Baldwin IT (2006) Novel rearranged abietane diterpenoids from the roots of Salvia sahendica. Chem Biodivers 3:916-922

Kintzios SE (2000) Sage: the genus salvia. Harwood Academic Publishers, Amsterdam, pp 55-68

Kolak U, Kabouche A, Öztürk M, Kabouche Z, Topçu G, Ulubelen A (2009) Antioxidant diterpenoids from the roots of Salvia barrelieri. Phytochem Anal 20(4):320-327

Kupchan SM, Karim A, Marcks C (1969) Tumor inhibitors. XLVIII. Taxodione and taxodone, two novel diterpenoid quinone methide tumor inhibitors from Taxodium distichum. J Org Chem 34(12):3912-3918

Kusumoto N, Ashitani T, Hayasaka Y, Murayama T, Ogiyama K, Takahashi K (2009) Antitermitic activities of abietane-type diterpenes from Taxodium distichum cones. J Chem Ecol 35(6):635-642

Machumi F, Samoylenko V, Yenesew A, Derese S, Midiwo JO, Wiggers FT, Jacob MR, Tekwani BL, Khan SI, Walker LA, Muhammad I (2010) Antimicrobial and antiparasitic abietane diterpenoids from the roots of Clerodendrum eriophyllum. Nat Prod Commun 5(6):853

Michavila A, Fernández-Gadea F, Rodríguez B (1985) Abietane diterpenoids from the root of Salvia lavandulaefolia. Phytochemistry 25(1):266-268
Michavila A, María C, Rodríguez B (1986) 20-Nor-abietane and rearranged abietane diterpenoids from the root of Salvia argentea. Phytochemistry 25(8):1935-1937

Moridi Farimani M, Mazarei Z (2014) Sesterterpenoids and other constituents from Salvia lachnocalyx Hedge. Fitoterapia 98:234-240

Moridi Farimani M, Miran M (2014) Labdane diterpenoids from Salvia reuterana. Phytochemistry 108:264-269

Moridi Farimani M, Taheri S, Matloubi Moghaddam F, Amin G (2008) Chemical constituents from Salvia macrosiphon Boiss. Chem Nat Comp 44:518-519

Moridi Farimani M, Matloubi Moghaddam F, Esmaeili MA, Amin GA (2012) Lupane triterpenoid and other constituents of Salvia eremophila. Nat Prod Res 26(21):2045-2049

Moridi Farimani M, Ebrahimi SN, Salehi P, Bahadori MB, Sonboli A, Khavasi HR, Zimmermann S, Kaiser M, Hamburger M (2013) Antitrypanosomal triterpenoid with an $\varepsilon$-lactone E-ring from Salvia urmiensis. J Nat Prod 76:1806-1809

Moujir L, Gutiérrez-Navarro AM, San Andrés L, Luis JG (1996) Bioactive diterpenoids isolated from Salvia mellifera. Phytother Res 10(2):172-174

Rechinger KH (1987) Flora Iranica. Akademische Druck- und Verlagsanstalt, Graz, pp 403-476

Saijo H, Kofujita H, Takahashi K, Ashitani T (2015) Antioxidant activity and mechanism of the abietane-type diterpene ferruginol. Nat Prod Res 29(18):1739-1743

Shaheen UY, Hussain MH, Ammar HA (2011) Cytotoxicity and antioxidant activity of new biologically active constituents from Salvia Lanigra and Salvia Splendens. Pharmacogn J 3(21):36-48

Tan N, Kaloga M, Radtke OA, Kiderlen AF, Öksüz S, Ulubelen A, Kolodziej H (2002) Abietane diterpenoids and triterpenoic acids from Salvia cilicica and their antileishmanial activities. Phytochemistry 61(8):881-884

Topçu G, Gören AC (2007) Biological activity of diterpenoids isolated from Anatolian Lamiaceae plants. Rec Nat Prod 1(1):1-16

Topcu G, Eriş C, Ulubelen A (1996) Rearranged abietane diterpenes from Salvia limbata. Phytochemistry 41(4):1143-1147

Topcu G, Kolak U, Ozturk M, Boga M, Hatipoglu SD, Bahadori F, Culhaoglu B, Dirmenci T (2013) Investigation of anticholinesterase activity of a series of Salvia extracts and the constituents of Salvia staminea. Nat Prod J 3(1):3-9

Ulubelen A (2003) Cardioactive and antibacterial terpenoids from some Salvia species. Phytochemistry 64(2):395-399

Ulubelen A, Topcu G, Tan N, Lin LJ, Cordell GA (1992) Microstegiol, a rearranged diterpene from Salvia microstegia. Phytochemistry 31(7):2419-2421

Ulubelen A, Topcu G, Eri C, Sönmez U, Kartal M, Kurucu S, Bozok-Johansson C (1994) Terpenoids from Salvia sclarea. Phytochemistry 36(4):971-974

Ulubelen A, Öksüz S, Kolak U, Tan N, Bozok-Johansson C, Çelik C, Kohlbau HJ, Voelter W (1999a) Diterpenoids from the roots of Salvia bracteata. Phytochemistry 52(8):1455-1459

Ulubelen A, Topçu G, Chai HB, Pezzuto JM (1999b) Cytotoxic activity of diterpenoids isolated from Salvia hypargeia. Pharm Boil 37(2):148-151

Ulubelen A, Oksüz S, Kolak U, Birman H, Voelter W (2000) Cardioactive terpenoids and a new rearranged diterpene from Salvia syriaca. Planta Med 66:627-629

Wu YB, Ni ZY, Shi QW, Dong M, Kiyota H, Gu YC, Cong B (2012) Constituents from Salvia species and their biological activities. Chem Rev 112:5967-6026

Yang Z, Kitano Y, Chiba K, Shibata N, Kurokawa H, Doi Y, Arakawa Y, Tada M (2001) Synthesis of variously oxidized abietane diterpenes and their antibacterial activities against MRSA and VRE. Bioorg Med Chem 9(2):347-356

Yong ZJH (1995) Two new diterpenoids, prtoketolactone and neoprionitone, from Salvia prionitis. Nat Prod Res Develop 4:1

Zaghloul AM, Gohar AA, Naiem ZAA, Bar FMA (2008) Taxodione, a DNAbinding compound from Taxodium distichum L. (Rich.). Z Naturforsch C 63(5-6):355-360 